\title{
Direction of Arrival Tracking of an Underwater Acoustic Source Using Particle Filtering: Real Data Experiments
}

\author{
Xionghu Zhong ${ }^{\star}$, A. B. Premkumar ${ }^{\star}$ and Wenwu Wang ${ }^{\dagger}$ \\ ${ }^{\star}$ School of Computer Engineering, College of Engineering, Nanyang Technological University, 639798, Singapore. \\ $\dagger$ CVSSP, Department of Electronic Engineering, University of Surrey, UK, GU2 7XH. \\ Email: ${ }^{\star}\{$ xhzhong, and asannamalai $\} @$ ntu.edu.sg, ${ }^{\dagger}$ w.wang@ surrey.ac.uk
}

\begin{abstract}
In the past, both theoretical work and practical implementation of particle filtering (PF) method have been extensively studied. However, its application in underwater signal processing has received much less attention. This paper intends to introduce $P F$ approach for underwater acoustic signal processing. Particularly, we are interested in direction of arrival (DOA) estimation using PF. A detailed introduction along with this perspective is presented in this paper. Since the noise usually spreads the mainlobe of likelihood function and causes problem in subsequent particle resampling step, an exponential weighted likelihood model is developed to emphasize particles at more relevant area. Hence, the the effect due to background noise can be reduced. Real underwater acoustic data collected in SWELLEx-96 experiment are employed to demonstrate the performance of the proposed PF approaches for underwater DOA tracking.
\end{abstract}

Index Terms-Direction of arrival, localization and tracking, underwater acoustics, particle filtering, SWELLEx-96 experiment.

\section{INTRODUCTION}

DOA estimation of an acoustic source in the underwater environment is an important topic and lies at the heart of many underwater signal processing applications such as sonar detection and localization [1]-[4], geoacoustic inversion [5], [6], underwater vehicle navigation [7], and ocean biological signal analysis [8]. It is a challenging problem since various uncertainties can arise from following aspects: 1) Source dynamics. Localization approaches usually assume that the source is static so that the source signal can be regarded as stationary. However, this is not realistic since the source may be moving around possibly with complicated trajectories particularly in a battle field. As such the received signal can no longer be assumed to be stationary and the traditional localization approaches degrade quickly; 2) Low signal-tonoise ration (SNR) of ocean noise environment. The received signals are distorted by ambient noise. Also, the noise process may be non-Gaussian and impulsive in nature; and 3) Shallow water environment. When a wave propagates in the shallow water environment, it can interact with the ocean surface and bottom, and results in dense dispersion due to the water medium and time varying (TV) characteristic of the ocean environment. The dispersive effect introduced by shallow water environments can severely degrade the performance of DOA estimation. Hence, directly applying existing approaches for DOA estimation in the underwater environment does not yield good estimates. It is important to develop advanced signal processing techniques which are able to take all these uncertainties into account.

Existing approaches for DOA estimation extensively rely on the localization techniques such as beamforming [9], [10], maximum likelihood [11], [12], MUSIC [13], and ESPRIT [14]. These approaches only employ the spatial information from current measurements. In the underwater applications, the DOAs are highly correlated in adjacent time steps. Hence, it is desired to exploit the information from both the previous DOA estimates and the current measurements to locate the source. In this paper, a Bayesian framework and its particle filtering implementation are introduced to cope with this problem. The particle filtering has been demonstrated to be an effective tool in coping with the versatile environments and motion uncertainties. At each time step, a number of particles are employed to represent the state and weighted to indicate its importance to the estimation. The selection scheme is then performed to replicate/discard the particles according to the high/low weight. Usually, the motion speed of an underwater target can be assumed slow. The motion dynamics can thus be modelled by using a constant velocity (CV) model. In [15], the conventional likelihood (CL) function is used to formulate the likelihood of the particles. The nuisance parameters such as source amplitude and noise variance are estimated by using a maximum likelihood estimator.

As we know, in the low SNR environments, the mainlobe of the likelihood function is usually spread, and spurious peaks are likely to be presented. This causes problem for using the conventional likelihood straightforwardly since the weights for the particles which are close to the ground truth are not emphasized enough with such a spread spectrum. The subsequent resampling scheme is thus very difficult to select the correct particles and replicate them. Given the particles drawn according to the $\mathrm{CV}$ model, the key task here is to design an efficient likelihood function which is able to enhance the particles from the source state area and reduce the 
effect from the noise. In this paper, a normalized likelihood function is constructed and it is further exponentially weighted to enhance the weight of the particles at high likelihood area. Real underwater data experiment studies show that the PF tracking algorithm with proposed enhanced likelihood model significantly outperforms the traditional localization approaches as well as the existing PF algorithm.

The rest of this paper is organized as follows. In Section II, the signal model and DOA estimation via localization approaches are introduced. Section III presents the source motion and likelihood models. The enhanced likelihood model and the tracking algorithm are also formulated. Real data experiments are organized in Section IV and conclusions are drawn in Section V.

\section{SignAl MOdEL AND LOCALIZATION FUNCTIONS}

Consider that a narrowband source signal $s(t)$ with known center frequency $f$ and the DOA location $\theta_{t}$ arriving on an array with $M$ elements at discrete time $t$. By using the complex envelop representation, the output of the sensor array can be written as

$$
\mathbf{y}(t)=\mathbf{a}\left(\theta_{t}\right) s(t)+\mathbf{n}(t),
$$

where $\mathbf{a}\left(\theta_{t}\right) \in \mathbb{C}^{M \times 1}$ is the steering vector toward the direction $\theta_{t}$ given as

$$
\mathbf{a}\left(\theta_{t}\right)=\left[1, e^{-j 2 \pi f \tau_{2}(t)}, \ldots, e^{-j 2 \pi f \tau_{M}(t)}\right]^{T},
$$

with $\tau_{m}(t)$, for $m=1, \ldots, M$ denoting the propagation delay between the first and the $m$ th sensor, and subscript $T$ representing the matrix transpose. For a uniform linear array with sensor separation $d$, the propagation delay $\tau_{m}(t)=$ $(m-1) d \cos \left(\theta_{t}\right) / c$ with $c$ denoting the sound propagation speed in the medium. $\mathbf{y}(t)=\left[y_{1}(t), \ldots, y_{M}(t)\right]^{T} \in \mathbb{C}^{M \times 1}$ is the vector of snapshots received by the sensor array. $\mathbf{n}(t)=$ $\left[n_{1}(t), \ldots, n_{M}(t)\right]^{T} \in \mathbb{C}^{M \times 1}$ is the additive noise process which is used to model the underwater noise environment. Since the source can be dynamic, the DOA $\theta_{t}$ itself is a random variable innovated as

$$
\theta_{t}=\theta_{t-1}+v_{t},
$$

where $v_{t}$ is the state noise process describing the innovation of the DOA $\theta_{t}$. The simplest choice for the PDF of the state noise process $v_{t}$ is a zero mean real Gaussian process, i.e., $v_{t} \sim \mathcal{N}\left(\cdot \mid 0, \sigma_{v}^{2}\right)$.

The objective of tracking is to estimate the DOA $\theta_{t}$ at different times $t$ based on the received data in (1) and the state transition model (3). Usually, following assumptions are made for the signal models:

A.1) The source signal $s(t)$ is a phase-modulated signal, i.e., $s(t)=a e^{j \phi_{t}}$. This means that the source signal has a constant amplitude $a$ and an independent and identically distributed (i.i.d.) random phase $\phi_{t}$, which is assumed to be uniformly distributed over $[0,2 \pi)$, i.e., $\phi_{t} \sim \mathcal{U}([0,2 \pi))$.
A.2) The noise term $n_{m}(t)$ is uncorrelated with the source signal $s(t)$ and statistically independent from sensor to sensor, i.e., $\mathbb{E}\left\{s(t) n_{m}^{*}(t)\right\}=0 ; \mathbb{E}\left\{n_{i}(t) n_{j}^{*}(t)\right\}=\sigma_{n}^{2} \delta_{i, j}$. Here $\mathbb{E}\{\cdot\}$ denotes the statistical expectation and superscript $*$ represents the conjugate operation. $\sigma_{n}^{2}$ is the variance of the noise process, and $\delta_{i, j}$ is Dirac-delta function with value 1 if $i=j$ and 0 otherwise.

A.3) The noise process $n_{m}(t)$ itself is a sequence of complexvalued i.i.d. circular Gaussian random variables with zero mean and covariance matrix $\sigma^{2}$, given as

$$
n_{m}(t) \sim \mathcal{C N}\left(\cdot \mid 0, \sigma^{2}\right) \quad \forall \quad m=1, \ldots, M
$$

where $\mathcal{C N}(\cdot \mid \mu, \Sigma)$ stands for the circular complex Gaussian distribution with mean $\mu$ and covariance $\Sigma$.

The assumptions A.1 and A.2 are general assumptions used in most array signal processing of underwater acoustics. Since the source signal is considered in the complex plane, the additive noise employed here is a complex-valued Gaussian process. This complex Gaussian process is further assumed to be circular in A.3 to guarantee that for a complex Gaussian variable $Z=X+j Y$, the variance of its real part $X$ and imaginary part $Y$ are the same and these two parts are uncorrelated.

Since underwater targets usually move slowly, the DOA $\theta_{t}$ can be assumed to stable if a small number of snapshots are processed at each time step. Assume that $L$ snapshots are taken into account at time step $k$, and let

$$
\mathbf{s}_{k}=[s(k L+1), \ldots, s(k L+L)],
$$

denote the snapshots of the source signal where $\mathbf{s}_{k} \in \mathbb{C}^{1 \times L}$. The noise and received data matrices can be expressed as

$$
\begin{aligned}
& \mathbf{N}_{k}=[\mathbf{n}(k L+1), \ldots, \mathbf{n}(k L+L)] ; \\
& \mathbf{Y}_{k}=[\mathbf{y}(k L+1), \ldots, \mathbf{y}(k L+L)],
\end{aligned}
$$

where $\mathbf{N}_{k}, \mathbf{Y}_{k} \in \mathbb{C}^{M \times L}$. Accordingly, $\theta_{k}$ is used to express the DOA at time step $k$.

Classical DOA estimation approaches such as Capon beamformer and subspace based methods extensively rely on the second order statistics of the received data matrix, i.e., the spatial covariance matrix. Based on the assumptions A.1 - A.3, the spatial covariance matrix can be written as

$$
\begin{aligned}
\mathbf{R}_{k} & \approx \mathbb{E}\left\{\mathbf{Y}_{k} \mathbf{Y}_{k}^{H}\right\} \\
& =\mathbf{a}\left(\theta_{k}\right) \mathbf{P}_{k} \mathbf{a}^{H}\left(\theta_{k}\right)+\sigma_{n}^{2} \mathbf{I}_{M},
\end{aligned}
$$

where $\mathbf{P}_{k}=\mathbb{E}\left\{\mathbf{s}_{k} \mathbf{s}_{k}^{H}\right\}$ is the covariance matrix of the source signal. The subscript $H$ denotes Hermitian transpose and $\mathbf{I}_{M}$ is an $M$ th order identity matrix.

The beamforming methods attempt to estimate the DOA by steering the array in the desired direction which results in maximum power output. The response of Bartlett beam forming and conventional likelihood are given respectively as 
[16]:

$$
\begin{aligned}
\mathcal{P}_{\text {Bartlett }}\left(\theta_{k}\right) & =\frac{\boldsymbol{a}^{H}(\theta) \mathbf{R}_{k} \boldsymbol{a}(\theta)}{\boldsymbol{a}^{H}(\theta) \boldsymbol{a}(\theta)}, \\
\mathcal{P}_{\mathrm{CL}}\left(\theta_{k}\right) & =\left\{\operatorname{tr}\left(\mathbf{R}_{k}\right)-\frac{\boldsymbol{a}^{H}\left(\boldsymbol{\theta}_{k}\right) \mathbf{R}_{k} \boldsymbol{a}\left(\boldsymbol{\theta}_{k}\right)}{\boldsymbol{a}^{H}\left(\boldsymbol{\theta}_{k}\right) \boldsymbol{a}\left(\boldsymbol{\theta}_{k}\right)}\right\}^{-M} .
\end{aligned}
$$

The DOA estimation can easily be obtained by implementing a one-dimensional search over the potential $\theta$ which maximizes the output of (9) or (10), given as

$$
\hat{\theta}_{k}=\arg \max _{0^{\circ} \leq \theta \leq 180^{\circ}} \mathcal{P}\left(\theta_{k}\right) .
$$

DOA estimation based on the localization approaches only use the spatial information from the current measurement sequence. Since the DOAs between adjacent time steps are highly correlated, it is desired to estimate the source DOA exploiting both the spatial and the temporal information (the latter is usually implied in the source dynamic model). In next section, the DOA estimation via Bayesian tracking is introduced.

\section{PARTICLE FILTERING FOR DOA TRACKING}

Consider that a source is currently at DOA $\theta_{k}$ and moving with a velocity $\dot{\theta}_{k}$ (in $\left.\mathrm{rad} / \mathrm{s}\right)$. The source state $\mathbf{x}_{k}$ can thus be constructed by the DOA $\theta_{k}$ and the motion velocity $\dot{\theta}_{k}$ given by $\mathbf{x}_{k}=\left[\theta_{k}, \dot{\theta}_{k}\right]^{T}$. Since the underwater target can usually be assumed moving slowly, the constant velocity (CV) model [17] that is different from equation (3) is used here, and is

$$
\mathbf{x}_{k}=\mathbf{A} \mathbf{x}_{k-1}+\mathbf{B} v_{k},
$$

where coefficient matrices $\mathbf{A}$ and $\mathbf{B}$ are defined by

$$
\mathbf{A}=\left[\begin{array}{cc}
1 & \Delta T \\
0 & 1
\end{array}\right], \quad \mathbf{B}=\left[\begin{array}{c}
\Delta T^{2} / 2 \\
\Delta T
\end{array}\right],
$$

with $\Delta T$ representing the time period in seconds between the previous and current time step, and $v_{k}$ is a zero-mean real Gaussian process (i.e., $v_{k} \sim \mathcal{N}\left(0, \sigma_{v}^{2}\right)$ ) used to model the turbulence on the source velocity.

The core step of applying a PF is to formulate the importance weight of each particle. At the last time step, assume that a set of particles $\mathbf{x}_{k}^{(i)}, i=1, \ldots, N$ with corresponding importance weight $w_{k}^{(i)}$ are available to approximate the posterior distribution of $p\left(\mathbf{x}_{k-1} \mid \mathbf{Y}_{1: k-1}\right)$. The particles are sampled at the current time step according to the source dynamic model (12), stated as

$$
\mathbf{x}_{k}^{(i)} \sim p\left(\mathbf{x}_{k}^{(i)} \mid \mathbf{x}_{k-1}^{(i)}\right) .
$$

The importance weights of the particles at current time step are given by

$$
w_{k}^{(i)}=w_{k-1}^{(i)} \frac{p\left(\mathbf{Y}_{k} \mid \mathbf{x}_{k}^{(i)}\right) p\left(\mathbf{x}_{k}^{(i)} \mid \mathbf{x}_{k-1}^{(i)}\right)}{q\left(\mathbf{x}_{k}^{(i)} \mid \mathbf{x}_{k-1}^{(i)}, \mathbf{Y}_{1: k-1}\right)},
$$

where $q(\cdot)$ stands for importance function. Since the particles are drawn according to the source dynamic model, we have

$$
q\left(\mathbf{x}_{k}^{(i)} \mid \mathbf{x}_{k-1}^{(i)}, \mathbf{Y}_{1: k-1}\right)=p\left(\mathbf{x}_{k}^{(i)} \mid \mathbf{x}_{k-1}^{(i)}\right) .
$$

The particles are thus weighted according to

$$
w_{k}^{(i)}=\tilde{w}_{k-1}^{(i)} p\left(\mathbf{Y}_{k} \mid \mathbf{x}_{k}^{(i)}\right),
$$

where $\tilde{w}_{k-1}^{(i)}$ is the normalized weight given as

$$
\tilde{w}_{k-1}^{(i)}=\frac{w_{k-1}^{(i)}}{\sum_{i=1}^{N} w_{k-1}^{(i)}} .
$$

After the resampling scheme, the posterior distribution of the state is thus

$$
p\left(\mathbf{x}_{k} \mid \mathbf{Y}_{1: k}\right) \approx \sum_{i=1}^{N} \tilde{w}_{k}^{(i)} \delta_{\mathbf{x}_{k}^{(i)}}\left(\mathbf{x}_{k}\right),
$$

where $\delta(\cdot)$ is a Dirac-delta function, and $N$ is the number of the particles. PF exploits the information from the source dynamic model as well as the current measurements. Since $\mathrm{PF}$ uses a number of particles to represent the source state, it admits uncertainties in the state-space model.

The likelihood function plays an important role in correcting the predicted states. It can be obtained according to the Bartlett beamforming response or conventional likelihood. As such the likelihood $p\left(\mathbf{Y}_{k} \mid \mathbf{x}_{k}\right)$ is the amplitude of the Bartlett or CL response, given by

$$
\begin{aligned}
& \hat{p}\left(\mathbf{Y}_{k} \mid \mathbf{x}_{k}\right)=\left|\mathcal{P}_{\text {Bartlett }}\left(\mathbf{C x}_{k}\right)\right|, \quad \text { or } \\
& \hat{p}\left(\mathbf{Y}_{k} \mid \mathbf{x}_{k}\right)=\left|\mathcal{P}_{\mathrm{CL}}\left(\mathbf{C x}_{k}\right)\right|,
\end{aligned}
$$

where $\mathbf{C}=[1,0]$ such that $\mathbf{C x}_{k}$ outputs the DOA $\theta_{k}$ of the state. This likelihood choice is actually a beamforming transform of the raw data. It is able to present the output of the state effectively. Given a state $\mathbf{C x}_{k}$, the Bartlett/CL response will be large if it is close to the ground truth and vice versa. The CL likelihood is employed for geoacoustic and source tracking problem in [15], [18] .

Due to the low SNR noisy environment, the mainlobe of the measurement function is usually spread and spurious peaks may appear. The Bartlett response and the conventional likelihood under the low SNR environment can be very flat. This causes problem for the subsequent step of calculating the weight of the particles. Generally, if the peak is very sharp and corresponds to the true DOA, the particles around the ground truth area can easily be resampled since they are weighted significantly more than the others. However, if the peak is spread or distorted, the particles cannot be weighted correctly by using such likelihood models. A new likelihood model which is able to emphasize the correct peak in the likelihood function should be developed.

Given a state $\mathbf{x}_{k}$, the aim here is to find a likelihood model which is able to reflect the importance of the particles and emphasize those close to the ground truth. To reshape the likelihood, the likelihood function is firstly normalized as

$$
\bar{p}\left(\mathbf{Y}_{k} \mid \mathbf{x}_{k}\right)=\frac{\hat{p}\left(\mathbf{Y}_{k} \mid \mathbf{x}_{k}\right)}{\max _{\mathbf{x}_{k}} \hat{p}\left(\mathbf{Y}_{k} \mid \mathbf{x}_{k}\right)} .
$$

It is further amended by an exponential weight, given as

$$
p\left(\mathbf{Y}_{k} \mid \mathbf{x}_{k}\right)=\left\{\bar{p}\left(\mathbf{Y}_{k} \mid \mathbf{x}_{k}\right)\right\}^{r}
$$


with $r \in \mathbb{R}^{+}$. After this weighting, the likelihood is reshaped and the particles located close to the ground truth can be enhanced. This step is very important since it is able to help the subsequent resampling algorithm to select and replicate the particles more efficiently. The PF algorithm introduced here is the same as that described in [19]. It is worth mentioning that the exponential exponent $r$ is determined by experimental study. By exponentially weighting the likelihood function properly, the particles in the highly likelihood area can be emphasized. However, an overly large $r$ can lead to an inappropriate emphasis of the particles, particularly if the likelihood function is distorted seriously by the noise.

\section{SWELLEX-96 EXPERIMENT}

The proposed PF tracking algorithms are further implemented by using the real experimental data recorded in SWellEx-96 event S5 [20]. Fig. 1 shows the experimental environment for the S5 event. The source started its track south of all of the arrays and proceeded northward at a speed of $2.5 \mathrm{~m} / \mathrm{s}$. Most of the source tow track occurred in water between $180 \mathrm{~m}$ and $220 \mathrm{~m}$ deep, with the second half of the tow along the $180 \mathrm{~m}$ isobath. The data recorded by the south horizontal linear arrays (HLA) equipped with 28 sensors are used. The HLA South array is a $255 \mathrm{~m}$ aperture horizontal array deployed on the seafloor. The line of bearing from the first element to the last element is -43.0 degree with respect to North. The data is recorded for 50 mins, with a sampling frequency of $3276.8 \mathrm{~Hz}$. The map of the array location is shown Fig. 1.

The data are resampled at $1000 \mathrm{~Hz}$ and first $5 \mathrm{~min}$ data are clipped to reduce the turbulence. Since the DOA of the source varies very slowly, the data is split into 45 time steps and different numbers of snapshots are employed to estimate

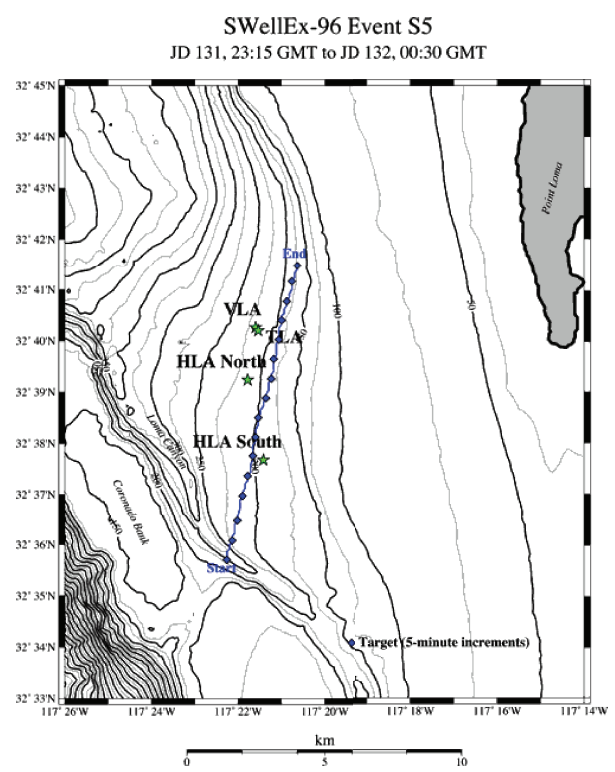

Fig. 1. Map of the real experiment setup. (Color version is available online at http://www.mpl.ucsd.edu/swellex96/s5.htm.)

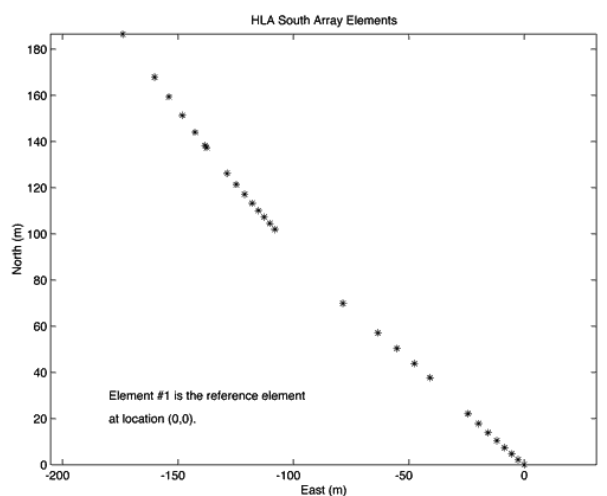

Fig. 2. Map of the array location. The HLA south array is not a uniform linear array but with a bow shape. The array is a $255 \mathrm{~m}$ aperture horizontal array deployed on the seafloor. The line of bearing from the first element to the last element is -43.0 degree with respect to North.

the DOA. The real ocean environment is found much more challenging than the simulated zero-mean Gaussian noisy environment. Hence, a large number of snapshots is thus used to achieve a satisfactory accuracy. In the experiment, we start from a processing length of $5 \mathrm{sec}$. since any length less than that is not able to present good results. The source moves at a velocity of 1 degree/sec. roughly. The details of environmental parameters such as sediment and water depth can be found online at http://www.mpl.ucsd.edu/swellex96/s5.htm.

Figure 3 presents the root mean square error (RMSE) versus different number of snapshots (i.e., different processing frame length). Five different approaches are implemented: CL based localization approach (CL), Bartlett beam forming based localization approach (Bartlett), CL based PF tracking approach (CL-PF), proposed exponentially weighted Bartlett likelihood based PF tracking approach (Bartlett-PF, $r=20$ ), and proposed exponentially weighted CL based PF tracking approach (CL-PF, $r=20$ ). It shows that the two proposed $\mathrm{PF}$ tracking approach with enhanced likelihood model is able to track the DOA of the source accurately. The proposed approaches significantly outperform the localization approaches and the existing particle filtering approach in [15]. It can also be observed that simply using the CL based PF algorithm here cannot track the DOA in the real ocean experiment; the performance is even worse than the localization approaches. This is because the noise due to ocean environment is very challenging and the $\mathrm{CL}$ is spread and distorted seriously. Similar estimation performance can also be found in probability of correct estimates (PROC) [19] as shown in Fig. 4. The DOA estimation accuracy can be enhanced significantly by using the proposed PF approaches.

\section{CONCLUSIONS}

A particle filtering approach is introduced in this paper to track an underwater acoustic source. The source motion uncertainty is modelled by using a CV model and the likelihood is formulated based on the Bartlett beamforming or conventional likelihood function. Unlike the existing approach which suffers 


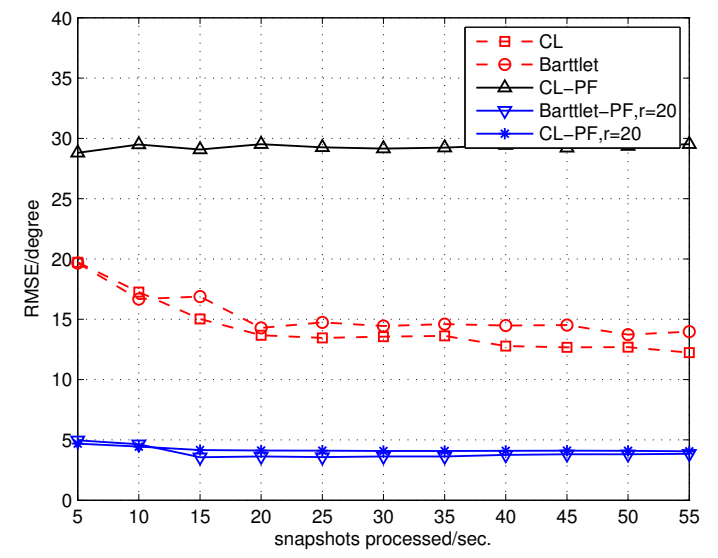

Fig. 3. RMSE vs. different number of snapshots for the real recorded data.

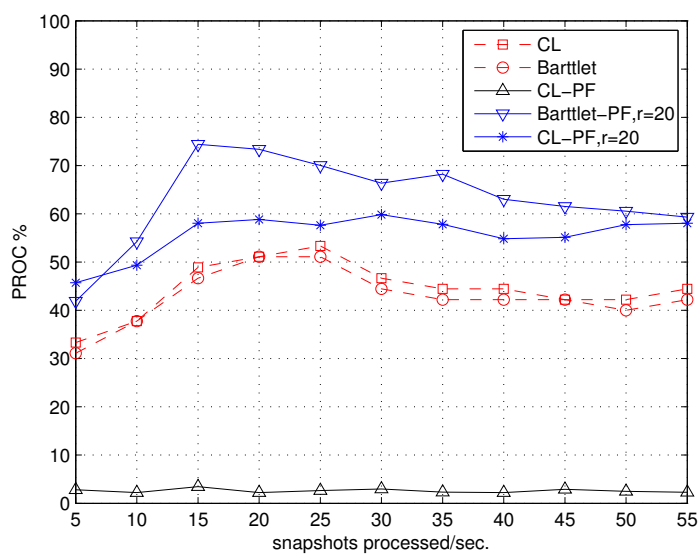

Fig. 4. PROC in percentage vs. different number of snapshots for the real recorded data.

from the heavy noise, the proposed PF tracking approaches are with an enhanced likelihood model which is able to emphasize the weight of particles at high likelihood area. Real underwater data experiments demonstrate that the proposed PF tracking approaches outperform the traditional localization approaches as well as the existing CL based PF tracking approach. In our future work, tracking (Bayesian) performance bound will be studied for underwater DOA estimation.

\section{REFERENCES}

[1] M. J. Hinich, "Maximum-likelihood signal processing for a vertical array," J. Acoust. Soc. Amer., vol. 54, no. 2, pp. 499-503, 1973.

[2] L. Bjorno, Ed., Underwater Acoustics and Signal Processing. Dordrecht: Reidel, 1981.

[3] A. B. Baggeroer, W. A. Kuperman, and P. N. Mikhalevsky, "An overview of matched field methods in ocean acoustics," IEEE J. Ocean. Eng., vol. 18 , no. 4, pp. 401-424, 1993.

[4] A. B. Gershman, V. I. Turchin, and V. A. Zverev, "Experimental results of localization of moving underwater signal by adaptive beamforming," IEEE Trans. Signal Process., vol. 43, no. 10, pp. 2249-2257, 1995.
[5] S. D. Rajan, J. F. Lynch, and G. V. Frisk, "Perturbative inversion methods for obtaining bottom geoacoustic parameters in shallow water," J. Acoust. Soc. Amer., vol. 82, no. 3, pp. 998-1017, 1987.

[6] P. A. Baxley, N. O. Booth, and W. S. Hodgkiss, "Matched-field replica model optimization and bottom property inversion in shallow water," $J$. Acoust. Soc. Amer., vol. 107, no. 3, pp. 1301-1323, 2000.

[7] V. Rigaud, L. Marce, J. L. Michel, and P. Borot, "Sensor fusion for auv localization," in Proc. (1990) Symp. Autonomous Underwater Vehicle Technology AUV '90, 1990, pp. 168-174.

[8] "Matched-field processing, geoacoustic inversion, and source signature recovery of blue whale vocalizations," J. Acoust. Soc. Amer., vol. 107, no. 3, pp. 1286-1300, Mar. 2000.

[9] M. S. Bartlett, "Smoothing periodograms from time series with continuous spectra," Nature, vol. 161, pp. 686-687, 1948.

[10] J. Capon, "High-resolution frequency-wavenumber spectrum ana," Proc. IEEE, vol. 57, no. 8, pp. 1408-1418, 1969.

[11] P. Stoica and A. Nehorai, "Performance study of conditional and unconditional direction-of-arrival estimation," Acoust., Speech and IEEE Trans. Signal Process., vol. 38, no. 10, pp. 1783-1795, 1990.

[12] B. Ottersten, M. Viberg, and T. Kailath, "Analysis of subspace fitting and $\mathrm{ml}$ techniques for parameter estimation from sensor array data," IEEE Trans. Signal Process., vol. 40, no. 3, pp. 590-600, 1992.

[13] R. O. Schmidt, "A signal subspace approach to multiple emitter location and spectral es," Ph.D. dissertation, Stanford Univ., Stanford, CA, Nov. 1981.

[14] R. Roy and T. Kailath, "Esprit-estimation of signal parameters via rotational invariance techniques," Acoust., Speech and IEEE Trans. Signal Process., vol. 37, no. 7, pp. 984-995, 1989.

[15] C. Yardim, P. Gerstoft, and W. S. Hodgkiss, "Geoacoustic and source tracking using particle filtering: experimental results," J. Acoust. Soc. Amer., vol. 128, no. 1, pp. 75-87, Jul. 2010.

[16] H. Krim and M. Viberg, "Two decades of array signal processing research: the parametric approach," IEEE Signal Process. Mag., vol. 13, no. 4, pp. 67-94, 1996.

[17] X. Rong Li and V. P. Jilkov, "Survey of maneuvering target tracking. part 1: Dynamic models," IEEE Trans. Aerosp. Electron. Syst., vol. 39, no. 4, pp. 1333-1364, Oct. 2003.

[18] C. Yardim, P. Gerstoft, and W. S. Hodgkiss, "Tracking of geoacoustic parameter using Kalman and particle filters," J. Acoust. Soc. Amer, vol. 125, no. 2, pp. 746-760, Feb. 2009.

[19] X. Zhong, V. N. Hari, A. Premkumar, and A. S. Madhukumar, "Particle filtering with enhanced likelihood model for underwater acoustic source DOA tracking," in OCEANS, 2011 IEEE - Spain, June 2011, pp. 1-6.

[20] J. Murray and D. Ensberg, "The swellex-96 experiment," May 1996. [Online]. Available: http://www.mpl.ucsd.edu/swellex96/ 